\title{
Examining the impact of social stressor stimuli in eliciting physiological reactivity in children and adolescents with autism spectrum disorder: $A$ systematic review and meta-analysis protocol
}

Agnes S. K. Wong ( $\nabla$ skagnes.wong@utoronto.ca )

University of Toronto Ontario Institute for Studies in Education https://orcid.org/0000-0002-7337-4148

Samantha Burns

University of Toronto Ontario Institute for Studies in Education

Earl Woodruff

University of Toronto Ontario Institute for Studies in Education

Protocol

Keywords: Autism spectrum disorder, Physiological reactivity, Social stressor stimuli, Children, Adolescents, Systematic review, Meta-analysis

Posted Date: September 20th, 2021

DOl: https://doi.org/10.21203/rs.3.rs-907926/v1

License: (c) (1) This work is licensed under a Creative Commons Attribution 4.0 International License.

Read Full License 


\section{Abstract}

Background: Stress is not experienced the same by everyone. Some individuals, such as individuals with Autism Spectrum Disorder (ASD), are at risk of heightened sensitivity to stress responses. ASD is a neurodevelopmental disorder commonly characterized by deficits in social communication and social interaction. Among different stressor stimuli, social stressors particularly worth our attention due to the social and communication challenges inherent to ASD. This study aims to systematically evaluate different social stressor stimuli in eliciting physiological reactivity in ASD, focusing on the children and adolescent population.

Methods: We designed a study protocol for this study and submitted it to PROSPERO for systematic review registration. Any studies with children and adolescents with ASD between the ages of 0-18 in clinical and community settings will be included. All types of social stressor interventions will be included. The intended outcomes will not be restrictive. The outcome of interest will include studies with physiological activity of the participants being measured, e.g., measures related to autonomic functioning, electrodermal functioning, and cortisol level. The primary literature sources will be across four electronic databases: Medline, Embase, PsycInfo, and CINAHL. The second source of literature will be across grey literature, including ProQuest Dissertations \& Theses Global and across clinical trial registries. Hand searching of references will be performed on the reference lists of all included studies. Two volunteers pursuing postgraduate-level studies will independently search and screen potential studies for eligibility. Finally, all references considered by hand-searching will be reviewed by two researchers. The methodological quality of the research will be assessed by adopting the quality assessment used by a previous study. The assessment consists of four primary categories: descriptive validity, internal validity, external validity, and statistical conclusion validity.

Discussion: Considering the inconsistent methodologies and findings in previous studies, a systematic review in this area is required. We are not aware of another systematic review discussing this specific issue. The findings will have important implications for clinical practice and research studies. We anticipate that the results will be of interest to multiple audiences, including the individuals with ASD, their families and caregivers, healthcare professionals, educators, and researchers.

\section{Systematic Review Registration: PROSPERO CRD42021244039}

\section{Introduction}

Stress can be defined as a real or perceived threat to an individual's physiological or psychological integrity (1). An optimal amount of stress leads to positive outcomes such as learning $(2,3)$ and health (4). However, outcomes of stress generally function as an inverted U-shaped curve $(2,3)$, where too high or too low a level of stress leads to detrimental consequences. For example, higher or lower than the optimal level of stress decreases memory (2). In addition, stress leads to different physiological responses such as changes in cardiac activity, perspiration, and skin temperature (5). Neurobiologically, 
stress leads to changes in the autonomic nervous system (ANS) and the hypothalamic-pituitary-adrenal axis (HPA axis) $(6,7)$. The ANS and HPA axis are essential regulators of modulating stress-related cardiovascular responsiveness and the vulnerability to stress-related disorders (8). For example, several studies have found elevated heart rate and cortisol level in participants following a high level of stress ( 9 , $10)$, and prolonged exposure to stress predisposes us to illness (11).

Research on stress impacts has primarily used experimental methods, such as providing participants with stimuli and recording the physiological responses, referred to as stressor stimuli. In this study, stressor stimuli refer to the situations hypothesized or intended to cause stress in the participants (12). Physiological reactivity refers to the difference between physiological activation in response to the stressor stimuli compared to the baseline level, which is commonly measured as the amount of activation in the ANS and HPA axis (12). The activation of the ANS is commonly measured by two categories of functioning: 1. autonomic functioning such as heart rate (HR), heart rate variability (HRV), respiratory sinus arrhythmia (RSA); and 2. electrodermal functioning such as electrodermal activity (EDA) or skin conductance level (SCL) (12-15). The HPA axis is measured largely through cortisol levels (12, 16). For example, using the blood draw stressor, participants were found to display elevated HR (17) by assessing the activation of the ANS and elevated cortisol level (18) by determining the HPA axis activation.

Stress is not homogeneous - i.e. not experienced the same by everyone. Some individuals are at risk of heightened sensitivity in their responses to stress. For example, individuals with Autism Spectrum Disorder (ASD) are particularly vulnerable as they have been found to exhibit heightened physiological reactivity to stress $(18,19)$. It requires our attention as heightened physiological reactivity to stress may increase the vulnerability to psychopathology $(20,21)$. ASD is a neurodevelopmental disorder commonly characterized by deficits in social communication and social interaction and the presence of restricted and repetitive patterns of behaviours and interests (22). For example, children with ASD were found to have significantly higher EDA during routine oral care than their TD peers (23), which correlated to their behavioural stress and anxiety, implying more physiological distress to these stressor stimuli in the ASD group. Among different stressor stimuli, social stressors, which include exposure to other individuals (i.e. through interactions or human images), cause more stress, particularly worth our attention due to the social and communication challenges inherent to ASD. Undoubtedly, social communication and interaction are unavoidable and crucial for employment and education. Several studies have found enhanced physiological responses in the ASD group using social stressor stimuli, such as significantly higher SCL (24) and cortisol level (25) than their typically developing peers. Another study which adopted the Trier Social Stress Test (TSST) found that children with ASD showed higher and sustained cortisol level (26). However, these findings are inconsistent. For example, some studies found blunted cortisol response (27) or no between-group differences in $\operatorname{EDA}(28,29), \mathrm{HR}$ and SCL $(30)$ in the ASD group even using similar social stressor stimuli. One potential mechanism for the lack of consistency is the heterogeneity with overall symptomatology that encompasses ASD (31-33). 
Due to the heterogeneity of ASD and the use of different physiological measures and different types of stressor stimuli across studies, the literature to date is characterized by inconsistent findings with an unclear magnitude of effects among other social stressor stimuli $(15,33,34)$. Given the importance attached to social communication and interaction and the fact that many social stressor stimuli are unavoidable, the atypical physiological reactivity to these stressors may create difficulties in everyday life and predispose to health problems in ASD. Considering the additional vulnerabilities and complexity experienced by people with ASD, findings must be integrated to inform further research and practice best. Therefore, this systematic review and meta-analysis aims to integrate evidence on the physiological response to social stressors stimuli in children and adolescents with ASD.

Considering the vulnerability to psychopathology among children and adolescents with ASD, the objective of this systematic review will be to investigate different stressor stimuli in eliciting physiological reactivity in ASD, with a particular focus on the children and adolescent population. Precisely, this review will aim to:

1. Identify and describe evidence that investigates the relationship between physiological responses and exposure to social stressor stimuli in children and adolescents with ASD.

2. Determine the strength of the evidence

\section{Methods}

\section{Protocol}

To integrate evidence across a range of sources with a common focus, this study protocol is part of an ongoing project that incorporates evidence across various methodological approaches to produce an extensive summary of the literature on physiological reactivity on social stressor stimuli in children and adolescents with ASD. Specifically, this review will assess the overall effect and strength of evidence on this topic. This integration of evidence will use principles of integrative reviews (35). This systematic review integrates similar conceptual and methodological studies.

This systematic review is reported following the guidance provided in the Preferred Reporting ltems for Systematic Reviews and Meta-Analyses Protocols (PRISMA-P) statement (36). (Additional file 1)

\section{Information source and literature search}

The primary literature sources will be across four electronic databases: Medline, Embase, Psyclnfo, and CINAHL. The second source of literature will be across grey literature, including ProQuest Dissertations \& Theses Global and across clinical trial registries. Hand searching of references will be performed on the reference lists of all included studies. An academic librarian will be consulted during the development of the search strategy. The search will consist of a broad range of terms and keywords related to ASD, social stressors, physiological responses, and children and adolescents (Additional file 2). One example of search syntax can be found in Additional file 3. 


\section{Eligibility criteria}

This study will follow the study design framework Population, Intervention, Comparator, Outcome(s) of interest (PICOS; (37)). The inclusion and exclusion criteria can be seen from Table 1.

1. Participants: We will include any studies with children and adolescents with ASD between the ages of 0-18 (38), of both genders, in both clinical and community settings (39), and from any country. Participants' intelligence levels will not be restricted.

2. Interventions and comparators: All types of social stressor interventions will be included if related to the eligibility criteria. As physiological reactivity refers to the difference between physiological activation in response to the stressor stimuli compared to the baseline level, the physiological reactivity will be examined by comparing it to the baseline level.

3. Outcome(s) of interest: The intended results will not be restrictive. The outcome of interest will include studies with physiological activity of the participants being measured, e.g., measures related to autonomic functioning (e.g., HR, HRV, and RSA), electrodermal functioning (e.g., EDA, SCL), and cortisol level.

\section{Table 1. Inclusion and Exclusion Criteria}

Inclusion

Any studies that include participants with ages between 0-18, inclusive

There are at least 1 participants diagnosed with ASD

Participants were exposed to at least one stressor stimulus condition different from the baseline

\section{Exclusion}

The publication was not in English

Only physiological activity at baseline was measured

The physiological measures were collected during challenging behaviours
The physiological activity of the participants was measured e.g., measures related to autonomic functioning (e.g., HR, HRV, and RSA), electrodermal functioning (e.g., EDA, SCL), or cortisol level.
The physiological measures were used for examining the treatment effects

The participants with different diagnoses were grouped together for analysis

Physiological responses to more than one stimulus were grouped together for analysis

\section{Selection and Screening Procedures}

After the instructions by two of the authors, two volunteers pursuing postgraduate level of studies will independently search and screen potential studies for eligibility. The first screening will be applied at a 
title and abstract level, based on the criteria specified above. The second will include screening at the fulltext level. Finally, all references considered by hand-searching will be reviewed by two researchers. A thirdparty senior researcher will resolve any disagreements. A Preferred Reporting Items for Systematic Reviews and Meta-Analyses (PRISMA) flow chart detailing studies included and excluded will be provided (36).

\section{Data collection}

Following screening and selection procedures of eligible articles, data extraction will occur using a predetermined template (Additional file 4). First, information on physiological outcome variables will be extracted. Next, social stressor stimuli information will be extracted based on whether the stimuli will be direct or indirect. Finally, demographic information will be collected and used as covariates in the pooled effect size calculations.

\section{Risk of bias and methodological quality assessment}

Considering the importance of the methodological quality of studies in affecting the interpretation of the systematic reviews $(40,41)$, the methodological quality of studies will be assessed. After careful consideration, the quality assessment used by a study (12), which was devised according to the methodological quality standards (40), was considered to be very suitable. Two independent raters will assess the methodological quality of the studies using the same criteria with minor adjustments. The assessment consists of 22 items in four main categories, including descriptive validity, internal validity, external validity, and statistical conclusion validity (12)

\section{Methods for evidence synthesis}

Regarding the approach in previous studies $(39,42)$, to calculate the effect size of different social stressor stimuli in eliciting physiological reactivity, the standardized mean difference effect size (Cohen's $d$ ) will be used to examine the differences in physiological measures before and after the exposure to stressor stimuli. In addition, the corrected standardized mean difference effect size (Hedges' $g$ ) will be used when the sample size is small.

Concerning the approach in other related studies (39), the Q statistical analysis will be used to examine whether the effect size variability across studies is more than the sampling error alone and then determine the use of a random-effects model or fixed-effects model. In addition, l-squared $(R)$ and Birgeratio tests will be used to examine heterogeneity and determine whether possible moderators should be tested subsequently (39).

\section{Additional analyses}

Possible moderators such as sample type, participants' age, gender, and intelligence level (if reported) will be examined if there are enough studies for each subgroup of stressor stimuli. Retrospective power analysis will be conducted for each moderator. 


\section{Bibliometric and Software considerations}

Identified records will be imported into Covidence, an online systematic review manager, where duplicates will be identified and removed. Bibliographic data management system (RefWorks) will be used to store and manage the results of the searches. To conduct the analyses, the R statistical software will be used.

\section{Ethics, Dissemination and Research Integrity}

No ethical approval is required for this study. Any amendments made to this protocol when conducting the study will be reported in the final manuscript. In addition, results will be disseminated through conferences and publications in relevant peer-reviewed journals.

\section{Patient and Public Involvement}

One of the study protocol co-authors (AW) offered occupational therapy training for children with ASD. Furthermore, we will evaluate whether the included studies had any patient or public involvement.

\section{Discussion}

Different social stressor stimuli and physiological measures were used across previous studies, and the results varied even when the same social stressor was used. Considering the inconsistent research methodologies and findings, a systematic review and meta-analysis study in this area is required. This study will identify and describe evidence that investigates the relationship between physiological responses and exposure to social stressor stimuli in children and adolescents with ASD and determine the strength of the evidence. To our current knowledge, there is not another systematic review and metaanalysis study discussing this specific issue. The findings will have important implications for clinical practice and future research studies. We anticipate that the results will be of interest to multiple audiences, including the individuals with ASD, their families and caregivers, healthcare professionals, educators, researchers, and scientists.

There are limitations of our planned, systematic review methods. For example, only studies written in English will be included, limiting the generalizability of the findings. Furthermore, this study explicitly examines the previous studies that adopted children and adolescents with ASD as participants, limiting the generalizability to the adult population. Despite the limitations, one of the important strengths of this study would be that it comprehensively and uniquely examines the impact of social stressor stimuli in eliciting physiological reactivity in children and adolescents with ASD, using both studies from the major databases as well as the grey literature. We anticipate that we will be able to identify the strength of the evidence among several commonly used social stressor stimuli and some moderators.

\section{Abbreviations}


ASD: Autism spectrum disorder; ANS: Autonomic nervous system; HPA axis: Hypothalamic-pituitaryadrenal axis; HR: Heart rate; HRV: Heart rate variability; RSA: Respiratory sinus arrhythmia; EDA: Electrodermal activity; SCL: Skin conductance level; PICOS: Population, Intervention, Comparator, Outcome(s) of interest; PRISMA: Preferred Reporting Items for Systematic Reviews and Meta-Analyses; PRISMA-P: Preferred Reporting Items for Systematic Review and Meta-Analysis extension for Protocols.

\section{Declarations}

\section{Acknowledgements}

Not applicable.

\section{Funding}

Not applicable.

\section{Availability of data and materials}

Not applicable.

\section{Ethical approval and consent to participate}

Not applicable.

\section{Competing interests}

The authors declare that they have no competing interests.

\section{Consent for publication}

Not applicable.

\section{Authors' contributions}

AW submitted this study for systematic review registration, provided the ideas and mainly contributed to the introduction and discussion sections, and edited the manuscript and additional files before submission. SB mainly contributed to the methods section in the first draft, and provided feedbacks on other sections. EW provided feedbacks and edited the whole manuscript before submission.

\section{Authors' information}

AW is a PhD student (Developmental Psychology and Education), M.A. (Developmental Psychology and Education), MOT (Occupational Therapy) and HKROT.

SB is a PhD student (Developmental Psychology and Education) and M.A. (Developmental Psychology and Education). 
EW is a PhD, and Chair at the Department of Applied Psychology and Human Development, Ontario Institute for Studies in Education, University of Toronto.

\section{Authors details}

${ }^{1}$ Ontario Institute for Studies in Education, University of Toronto, 252 Bloor St W. Toronto, ON M5S 1V6, Canada

\section{References}

(1) McEwen BS. The neurobiology of stress: from serendipity to clinical relevance. Brain Res 2000;886(12):172-189. https://doi.org/10.1016/s0006-8993(00)02950-4

(2) Salehi B, Cordero MI, Sandi C. Learning under stress: the inverted-U-shape function revisited. Learn Mem 2010 Sep 30;17(10):522-530. https://doi.org/10.1101/Im.1914110

(3) Rudland JR, Golding C, Wilkinson TJ. The stress paradox: How stress can be good for learning. Med Educ 2020 Jan;54(1):40-45. https://doi.org/10.1111/medu.13830

(4) Zastrow C. How to manage stress. Indian Journal of Social Work 1984;44(4):365-375.

(5) Kushki A, Drumm E, Pla Mobarak M, Tanel N, Dupuis A, Chau T, et al. Investigating the autonomic nervous system response to anxiety in children with autism spectrum disorders. PLoS One 2013;8(4):e59730. https://doi.org/10.1371/journal.pone.0059730

(6) Levine S. Developmental determinants of sensitivity and resistance to stress.

Psychoneuroendocrinology 2005;30(10):939-946. https://doi.org/10.1016/j.psyneuen.2005.03.013

(7) Kemeny ME. The psychobiology of stress. Current directions in psychological science 2003;12(4):124129.

(8) Agorastos A, Heinig A, Stiedl O, Hager T, Sommer A, Muller JC, et al. Vagal effects of endocrine HPA axis challenges on resting autonomic activity assessed by heart rate variability measures in healthy humans. Psychoneuroendocrinology 2019 Apr;102:196-203.

https://doi.org/10.1016/j.psyneuen.2018.12.017

(9) Ditzen B, Neumann ID, Bodenmann G, von Dawans B, Turner RA, Ehlert U, et al. Effects of different kinds of couple interaction on cortisol and heart rate responses to stress in women.

Psychoneuroendocrinology 2007;32(5):565-574. https://doi.org/10.1016/j.psyneuen.2007.03.011

(10) Reinhardt T, Schmahl C, Wust S, Bohus M. Salivary cortisol, heart rate, electrodermal activity and subjective stress responses to the Mannheim Multicomponent Stress Test (MMST). Psychiatry Res 2012 Jun 30;198(1):106-111. https://doi.org/10.1016/j.psychres.2011.12.009 
(11) Khansari DN, Murgo AJ, Faith RE. Effects of stress on the immune system. Immunol Today 1990;11:170-175. https://doi.org/10.1016/0167-5699(90)90069-I

(12) Lydon S, Healy O, Reed P, Mulhern T, Hughes BM, Goodwin MS. A systematic review of physiological reactivity to stimuli in autism. Dev Neurorehabil 2016 Dec;19(6):335-355.

https://doi.org/10.3109/17518423.2014.971975

(13) Cuve HC, Gao Y, Fuse A. Is it avoidance or hypoarousal? A systematic review of emotion recognition, eye-tracking, and psychophysiological studies in young adults with autism spectrum conditions. Res Autism Spectr Disord 2018;55:1-13. https://doi.org/10.1016/j.rasd.2018.07.002

(14) Stroud LR, Foster E, Papandonatos GD, Handwerger K, Granger DA, Kivlighan KT, et al. Stress response and the adolescent transition: performance versus peer rejection stressors. Dev Psychopathol 2009 Winter;21(1):47-68. https://doi.org/10.1017/S0954579409000042

(15) Benevides TW, Lane SJ. A review of cardiac autonomic measures: considerations for examination of physiological response in children with autism spectrum disorder. J Autism Dev Disord 2015 Feb;45(2):560-575. https://doi.org/10.1007/s10803-013-1971-z

(16) Smeekens I, Didden R, Verhoeven EW. Exploring the relationship of autonomic and endocrine activity with social functioning in adults with autism spectrum disorders. J Autism Dev Disord 2015 Feb;45(2):495-505. https://doi.org/10.1007/s10803-013-1947-z

(17) Tordjman S, Anderson GM, Botbol M, Brailly-Tabard S, Perez-Diaz F, Graignic R, et al. Pain reactivity and plasma beta-endorphin in children and adolescents with autistic disorder. PLoS One 2009 Aug 26;4(8):e5289. https://doi.org/10.1371/journal.pone.0005289

(18) Spratt EG, Nicholas JS, Brady KT, Carpenter LA, Hatcher CR, Meekins KA, et al. Enhanced cortisol response to stress in children in autism. J Autism Dev Disord 2012 Jan;42(1):75-81. https://doi.org/10.1007/s10803-011-1214-0

(19) Taylor JL, Corbett BA. A review of rhythm and responsiveness of cortisol in individuals with autism spectrum disorders. Psychoneuroendocrinology 2014;49:207-228.

https://doi.org/10.1016/j.psyneuen.2014.07.015

(20) Spear LP. Heightened stress responsivity and emotional reactivity during pubertal maturation: Implications for psychopathology. Dev Psychopathol 2009 Winter;21(1):87-97. https://doi.org/10.1017/S0954579409000066

(21) Corbett BA, Simon D. Adolescence, Stress and Cortisol in Autism Spectrum Disorders. OA Autism 2014 Feb 1;1(1):2.

(22) American Psychiatric Association. Diagnostic and statistical manual of mental disorders (DSM-5®). : American Psychiatric Pub; 2013. 
(23) Stein LI, Lane CJ, Williams ME, Dawson ME, Polido JC, Cermak SA. Physiological and behavioral stress and anxiety in children with autism spectrum disorders during routine oral care. Biomed Res Int 2014;2014:694876. https://doi.org/10.1155/2014/694876

(24) Joseph RM, Ehrman K, McNally R, Keehn B. Affective response to eye contact and face recognition ability in children with ASD. Journal of the International Neuropsychological Society 2008;14(6):947-955. https://doi.org/10.1017/S1355617708081344

(25) Schupp CW, Simon D, Corbett BA. Cortisol responsivity differences in children with autism spectrum disorders during free and cooperative play. J Autism Dev Disord 2013 Oct;43(10):2405-2417. https://doi.org/10.1007/s10803-013-1790-2

(26) Corbett BA, Schupp CW, Lanni KE. Comparing biobehavioral profiles across two social stress paradigms in children with and without autism spectrum disorders. Mol Autism 2012 Nov 17;3(1):132392-3-13. https://doi.org/10.1186/2040-2392-3-13

(27) Edmiston EK, Blain SD, Corbett BA. Salivary cortisol and behavioral response to social evaluative threat in adolescents with autism spectrum disorder. Autism Res 2017 Feb;10(2):346-358. https://doi.org/10.1002/aur.1660

(28) Levine TP, Sheinkopf SJ, Pescosolido M, Rodino A, Elia G, Lester B. Physiologic arousal to social stress in children with autism spectrum disorders: A pilot study. Res Autism Spectr Disord 2012 Winter;6(1):177-183. https://doi.org/10.1016/j.rasd.2011.04.003

(29) Kaartinen M, Puura K, Makela T, Rannisto M, Lemponen R, Helminen M, et al. Autonomic arousal to direct gaze correlates with social impairments among children with ASD. J Autism Dev Disord 2012 Sep;42(9):1917-1927. https://doi.org/10.1007/s10803-011-1435-2

(30) Louwerse A, van Der Geest J, Tulen J, van der Ende J, Van Gool A, Verhulst F, et al. Effects of eye gaze directions of facial images on looking behaviour and autonomic responses in adolescents with autism spectrum disorders. Res Autism Spectr Disord 2013;7(9):1043-1053. https://doi.org/10.1016/j.rasd.2013.04.013

(31) Rice K, Moriuchi JM, Jones W, Klin A. Parsing heterogeneity in autism spectrum disorders: Visual scanning of dynamic social scenes in school-aged children. Journal of the American Academy of Child \& Adolescent Psychiatry 2012;51(3):238-248. https://doi.org/10.1016/j.jaac.2011.12.017

(32) Louwerse A, Tulen JH, van der Geest JN, van der Ende J, Verhulst FC, Greaves-Lord K. Autonomic responses to social and nonsocial pictures in adolescents with autism spectrum disorder. Autism Res 2014 Feb;7(1):17-27. https://doi.org/10.1002/aur.1327

(33) Ellenbroek BA, Sengul HK. Autism spectrum disorders: Autonomic alterations with a special focus on the heart. Heart and Mind 2017;1(2):78. https://doi.org/10.4103/hm.hm_5_17 
(34) Corbett BA, Muscatello RA, Kim A, Patel K, Vandekar S. Developmental effects in physiological stress in early adolescents with and without autism spectrum disorder. Psychoneuroendocrinology 2021 Mar;125:105115. https://doi.org/10.1016/j.psyneuen.2020.105115

(35) Whittemore R, Knafl K. The integrative review: Updated methodology. J Adv Nurs 2005;52(5):546553. https://doi.org/10.1111/j.1365-2648.2005.03621.x

(36) Page MJ, McKenzie JE, Bossuyt PM, Boutron I, Hoffmann TC, Mulrow CD, et al. The PRISMA 2020 statement: An updated guideline for reporting systematic reviews. J Clin Epidemiol 2021 Mar 17. https://doi.org/10.1136/bmj.n71

(37) Joanna Briggs Institute. Joanna Briggs Institute Reviewers' Manual: 2014 edition Australia: The Joanna Briggs Institute.

(38) Crane S, Broome ME. Understanding ethical issues of research participation from the perspective of participating children and adolescents: A systematic review. Worldviews Evid Based Nurs 2017 Jun;14(3):200-209. https://doi.org/10.1111/wvn.12209

(39) Seddon JA, Rodriguez VJ, Provencher Y, Raftery-Helmer J, Hersh J, Labelle PR, et al. Meta-analysis of the effectiveness of the Trier Social Stress Test in eliciting physiological stress responses in children and adolescents. Psychoneuroendocrinology 2020 Jun;116:104582.

https://doi.org/10.1016/j.psyneuen.2020.104582

(40) Farrington DP. Methodological quality standards for evaluation research. Ann Am Acad Pol Soc Sci 2003;587(1):49-68. https://doi.org/10.1177/0002716202250789

(41) Moher D, Shamseer L, Clarke M, Ghersi D, Liberati A, Petticrew M, et al. Preferred Reporting Items for Systematic Review and Meta-Analysis Protocols (PRISMA-P) 2015 statement. Syst Rev. 2015;4(1). https://doi.org/10.1186/2046-4053-4-1

(42) Frazier TW, Strauss M, Klingemier EW, Zetzer EE, Hardan AY, Eng C, et al. A meta-analysis of gaze differences to social and nonsocial information between individuals with and without autism. J Am Acad Child Adolesc Psychiatry 2017 Jul;56(7):546-555. https://doi.org/10.1016/j.jaac.2017.05.005

\section{Supplementary Files}

This is a list of supplementary files associated with this preprint. Click to download.

- Additionalfile1PRISMAPChecklist.docx

- Additionalfile2KeySearchTerms.docx

- Additionalfile3SearchSyntaxExamplePsycinfo.docx

- Additionalfile4PredeterminedDataExtractionTemplate.docx 